\title{
Spin-Orbit Interaction in Symmetric Wells with Two Subbands
}

\author{
Esmerindo Bernardes, ${ }^{1}$ John Schliemann, ${ }^{2,3}$ Minchul Lee, ${ }^{3}$ J. Carlos Egues,,${ }^{1,3,4}$ and Daniel Loss ${ }^{3,4}$ \\ ${ }^{1}$ Instituto de Física de São Carlos, Universidade de São Paulo, 13560-970 São Carlos, São Paulo, Brazil \\ ${ }^{2}$ Institute for Theoretical Physics, University of Regensburg, D-93040 Regensburg, Germany \\ ${ }^{3}$ Department of Physics and Astronomy, University of Basel, CH-4056 Basel, Switzerland \\ ${ }^{4}$ Kavli Institute for Theoretical Physics, University of California, Santa Barbara, California 93106, USA
}

(Received 11 July 2006; published 17 August 2007)

\begin{abstract}
We investigate the spin-orbit (SO) interaction in two-dimensional electron gases in quantum wells with two subbands. From the $8 \times 8 \mathrm{Kane}$ model, we derive a new intersubband-induced SO term which resembles the functional form of the Rashba SO but is nonzero even in symmetric structures. This follows from the distinct parity of the confined states (even or odd) which obliterates the need for asymmetric potentials. We self-consistently calculate the new SO coupling strength for realistic wells and find it comparable to the usual Rashba constant. Our new SO term gives rise to a nonzero ballistic spin-Hall conductivity, which changes sign as a function of the Fermi energy $\left(\varepsilon_{F}\right)$ and can induce an unusual Zitterbewegung with cycloidal trajectories without magnetic fields.
\end{abstract}

DOI: 10.1103/PhysRevLett.99.076603

The rapidly developing field of spintronics has generated a great deal of interest in spin-orbit (SO) coupling in semiconductor nanostructures [1]. For an $n$-doped zincblende semiconductor quantum well with only the lowest subband occupied, i.e., in a strictly $2 \mathrm{D}$ situation, there are two main contributions to the interaction of the spin and orbital degrees of freedom of electrons. One contribution is the Dresselhaus term, which results from the lack of inversion symmetry of the underlying zinc-blende lattice [2] and is to lowest order linear in the crystal momentum [3]. This linearity is shared by the other contribution known as the Rashba term [4], which is due to structural inversion asymmetry and can be tuned by an electric gate across the well [5]. These two contributions can lead to an interesting interplay in spintronic systems [6].

In this Letter we consider yet another type of electronic SO coupling which, as we show, occurs in III-V (or II-VI) zinc-blende semiconductor quantum wells with more than one subband. We derive a new intersubband-induced SO interaction which resembles that of the ordinary Rashba model; however, in contrast to the latter, ours is nonzero even in symmetric structures (Fig. 1). We self-consistently determine the strength of this new SO coupling for realistic single and double wells and find it comparable to the Rashba constant [Figs. 2(a) and 2(b)]. We have investigated the spin-Hall effect and the dynamics of spinpolarized electrons due to this new SO term. We find (i) a nonzero ballistic spin-Hall conductivity which changes sign as a function of $\varepsilon_{F}$ and (ii) an unusual Zitterbewegung [7] with cycloidal trajectories without magnetic fields (Fig. 3). As derived below, for a symmetric well with two subbands our $4 \times 4$ electron Hamiltonian is

$$
\begin{aligned}
\mathcal{H}= & \left(\frac{\vec{p}^{2}}{2 m^{*}}+\boldsymbol{\epsilon}_{+}\right) \mathbf{1} \otimes \mathbf{1}-\boldsymbol{\epsilon}_{-} \tau^{z} \otimes \mathbf{1} \\
& +\frac{\eta}{\hbar} \tau^{x} \otimes\left(p_{x} \sigma^{y}-p_{y} \sigma^{x}\right),
\end{aligned}
$$

PACS numbers: 72.25.Dc, 71.70.Ej, 73.21.Fg, 85.75.-d

where $m^{*}$ is the effective mass, $\epsilon_{ \pm}=\left(\varepsilon_{o} \pm \varepsilon_{e}\right) / 2, \varepsilon_{e}$ and $\varepsilon_{o}$ are quantized energies of the lowest (even) and first excited (odd) subbands (corresponding to eigenstates $|e\rangle$ and $|o\rangle$ ), respectively, measured from the bottom of the quantum well, $\tau^{x, y, z}$ denote the Pauli matrices describing the subband (or pseudospin) degree of freedom, and $\sigma^{x, y, z}$ are Pauli matrices referring to the electron spin. The new intersubband-induced SO coupling $\eta$ is

$$
\begin{aligned}
\eta= & -\left(\frac{1}{E_{g}^{2}}-\frac{1}{\left(E_{g}+\Delta\right)^{2}}\right) \frac{P^{2}}{3}\left\langle e\left|\partial_{z} V(z)\right| o\right\rangle \\
& +\left(\frac{\delta_{v}}{E_{g}^{2}}-\frac{\delta_{\Delta}}{\left(E_{g}+\Delta\right)^{2}}\right) \frac{P^{2}}{3}\left\langle e\left|\partial_{z} h(z)\right| o\right\rangle,
\end{aligned}
$$

where $E_{g}$ and $\Delta$ are the fundamental and split-off band gaps in the well region [8], $P$ is the Kane matrix element [9]. The parameters $\delta_{v}$ and $\delta_{\Delta}$ denote valence-band offsets between the well and the barrier regions [10], $V(z)$ is the Hartree-type contribution to the electron potential, and $h(z)$ is the structural quantum-well profile [11]. Note that

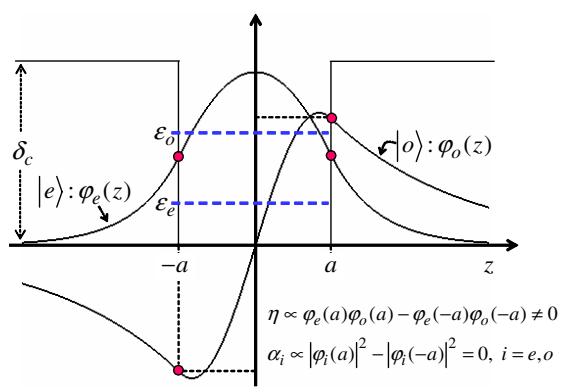

FIG. 1 (color online). Square well with its ground-state $\varphi_{e}(z)$ and first excited-state $\varphi_{o}(z)$ wave functions. The new intersubband-induced SO coupling $\eta$ in Eq. (4) is nonzero even in symmetric wells due to the distinct parities of $\varphi_{e}(z)$ (even) and $\varphi_{o}(z)$ (odd), which yield a nonvanishing matrix element for the derivative of the symmetric potential. 

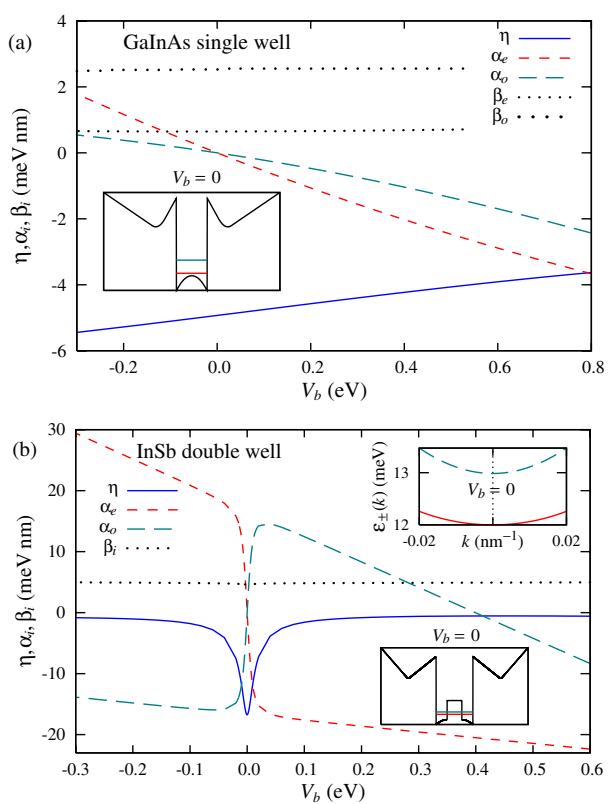

FIG. 2 (color online). Calculated SO coupling strengths as a function of the external gate $V_{b}$ for realistic wells. (a) For the single GaInAs [16,20] well studied, the intersubband coupling $\eta$ is larger than the Dresselhaus $\beta_{i}$ and the Rashba $\alpha_{i}$ constants $(i=e, o)$. Note that $\left|\alpha_{e}\right| \geq\left|\alpha_{o}\right|$ and both change sign across $V_{b}=0$ (in contrast to $\beta_{i}$ and $\eta$ ). (b) For the InSb double well considered, $\eta$ shows a "resonant behavior" about $V_{b}=0$ [symmetric configuration, lower-left inset in (b)]. This occurs because the subband splitting $\varepsilon_{o}-\varepsilon_{e}$ reaches a minimum at $V_{b}=0$ and the double-well wave functions are very similar (though of distinct parities) for $V_{b} \sim 0$. This also makes $\alpha_{e} \sim-\alpha_{o}$ around $V_{b}=0$. Upper-right inset in (b): Energy dispersions $\varepsilon_{ \pm}(\vec{k})$ [Eq. (10)] of the symmetric double well.

$\eta$ can be varied via external gates (Fig. 2). Next we outline the derivation of $\mathcal{H}$ in Eq. (1).

Kane Hamiltonian. -We start from the usual $8 \times 8$ Kane Hamiltonian describing the $s$-type conduction and the $p$-type valence bands around the $\Gamma$ point [12],

$$
\mathcal{H}_{8 \times 8}=\left(\begin{array}{cc}
H_{c} & H_{c v} \\
H_{v c} & H_{v}
\end{array}\right),
$$

where $H_{c}$ is a $2 \times 2$ diagonal matrix with elements

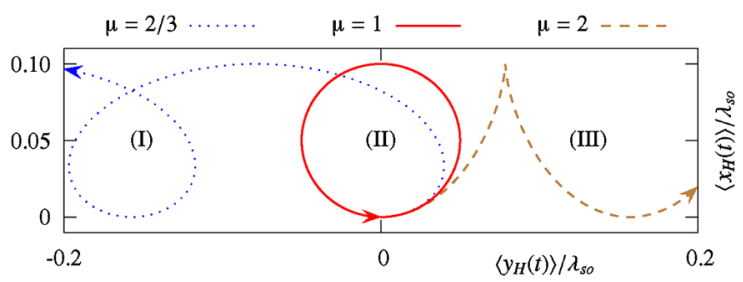

FIG. 3 (color online). Zitterbewegung due to the SO coupling $\eta$ for distinct ratios $\mu=\epsilon_{-} / 2 \varepsilon_{\text {SO }}$. Note the peculiar trajectories with the forward injected electrons moving backward (I) and even in a closed path (II). This follows from the SO induced change in the curvature of the bands which renormalizes the effective masses. Here we use $\lambda_{\mathrm{SO}} k_{0 y}=\mu / 10, \lambda_{\mathrm{SO}}^{-1}=m^{*} \eta / \hbar$. $p^{2} / 2 m_{0}+V_{c}(\vec{r}), m_{0}$ is the bare electron mass, $H_{v}$ is a $6 \times$ 6 diagonal matrix with elements $p^{2} / 2 m_{0}+V_{v}(\vec{r})-E_{g}$ for the heavy- and light-hole bands, and $p^{2} / 2 m_{0}+V_{\Delta}(\vec{r})-$ $E_{g}-\Delta$ for the split-off band, $V_{i}(\vec{r})(i=c, v, \Delta)$ denote arbitrary potentials (see below), and $H_{c v}=\left(H_{v c}\right)^{\dagger}$ is

$$
H_{c v}=\left(\begin{array}{cccccc}
\frac{-\kappa_{+}}{\sqrt{2}} & \sqrt{\frac{2}{3}} \kappa_{z} & \frac{\kappa_{-}}{\sqrt{6}} & 0 & \frac{-\kappa_{z}}{\sqrt{3}} & \frac{-\kappa_{-}}{\sqrt{3}} \\
0 & \frac{-\kappa_{+}}{\sqrt{6}} & \sqrt{\frac{2}{3}} \kappa_{z} & \frac{\kappa_{-}}{\sqrt{2}} & \frac{-\kappa_{+}}{\sqrt{3}} & \frac{\kappa_{z}}{\sqrt{3}}
\end{array}\right),
$$

where $\vec{\kappa}=P \vec{k}, \vec{k}=\vec{p} / \hbar$ is the electron wave vector, $k_{ \pm}=$ $k_{x} \pm i k_{y}$, and $P=-i \hbar\left\langle S\left|p_{x}\right| X\right\rangle / m_{0}$ parametrizes the conduction-to-valence-band coupling, $|S\rangle$ and $|X\rangle$ are the usual periodic Bloch functions at the $\Gamma$ point.

Effective electron Hamiltonian: Folding down.-The Kane Hamiltonian (3) acts on an eight-component spinor $\Psi^{\dagger}=\left(\begin{array}{ll}\psi_{c} & \psi_{v}\end{array}\right)^{\dagger}$ in which the last six components $\psi_{v}$ represent valence-band states. By eliminating the hole components from the Schrödinger equation $\mathcal{H}_{8 \times 8} \Psi=$ $\varepsilon \Psi$, where $\varepsilon$ is the eigenenergy, we can fold down this $8 \times$ 8 equation into a $2 \times 2$ effective equation for the conduction-band states only: $\mathcal{H}(\varepsilon) \tilde{\psi}_{c}=\left[H_{c}+H_{c v}(\varepsilon-\right.$ $\left.\left.H_{v}\right)^{-1} H_{v c}\right] \tilde{\psi}_{c}, \tilde{\psi}_{c}$ is a renormalized conduction-electron spinor.

SO in symmetric wells. - Applying the above procedure to a quantum well, defined by the confining potentials [11] $V_{i}(\vec{r}) \rightarrow V_{i}(z)=V(z)+\delta_{i} h(z), i=c, v, \Delta$, we find

$$
\begin{gathered}
\mathcal{H}(\varepsilon)=H_{\mathrm{QW}}+\frac{P^{2}}{3 \hbar^{2}} p_{-}\left[\eta_{1}^{-1}+\eta_{2}^{-1}, p_{z}\right], \\
H_{\mathrm{QW}}=p_{+} \frac{1}{2 m^{*}(z, \varepsilon)} p_{-}+p_{z} \frac{1}{2 m^{*}(z, \varepsilon)} p_{z}+V_{c}(z),
\end{gathered}
$$

where $1 / m^{*}(z, \varepsilon)=\left(2 P^{2} / 3 \hbar^{2}\right)\left(2 / \eta_{1}+1 / \eta_{2}\right)+1 / m_{0}$, $\eta_{1}=\varepsilon-\left[p^{2} / 2 m_{0}+V(z)-\delta_{v} h(z)-E_{g}\right]$ and $\eta_{2}=$ $\varepsilon-\left[p^{2} / 2 m_{0}+V(z)-\delta_{\Delta} h(z)-E_{g}-\Delta\right]$. Equation (5) describes an electron in a quantum well $\left(H_{\mathrm{QW}}\right.$ term) with spin-orbit interaction (last term) [13]. The kinetic-energy operators above are complicated due to the position- and energy-dependent effective mass $m^{*}(z, \varepsilon)$. Since $E_{g}$ and $E_{g}+\Delta$ are the largest energy scales in our system, we can simplify (5) and (6) by expanding $1 / \eta_{1}$ and $1 / \eta_{2}$ in the form $1 / \eta_{1}=E_{g}^{-1}\left\{1-\left[\varepsilon-p^{2} / 2 m_{0}-V(z)+\delta_{v} h(z)\right] /\right.$ $\left.E_{g}+\cdots\right\}$ and $1 / \eta_{2}=\left(E_{g}+\Delta\right)^{-1}\left\{1-\left[\varepsilon-p^{2} / 2 m_{0}-\right.\right.$ $\left.\left.V(z)+\delta_{\Delta} h(z)\right] /\left(E_{g}+\Delta\right)+\cdots\right\}$. To zeroth order $\eta_{1}=$ $E_{g}, \quad \eta_{2}=E_{g}+\Delta, \quad$ and $\quad H_{\mathrm{QW}}=p_{\|}^{2} / 2 m^{*}+p_{z}^{2} / 2 m^{*}+$ $V_{c}(z)$ with (a constant effective mass) $1 / m^{*}=$ $\left(2 P^{2} / 3 \hbar^{2}\right)\left[2 / E_{g}+1 /\left(E_{g}+\Delta\right)\right]+1 / m_{0}$ [14]. Since the SO operator $\left[\eta_{1}^{-1}+\eta_{2}^{-1}, p_{z}\right] \rightarrow \partial_{z}\left(1 / \eta_{1}\right)+\partial_{z}\left(1 / \eta_{2}\right)$, we need to keep the first-order terms in the expansions of $\eta_{1}^{-1}$ and $\eta_{2}^{-1}$ which yield the leading nonzero contribution to the SO term in (5). We find $\left[\eta_{1}^{-1}+\eta_{2}^{-1}, p_{z}\right]=\left[1 / E_{g}^{2}-\right.$ $\left.1 /\left(E_{g}+\Delta\right)^{2}\right] \partial_{z} V(z)-\left[\delta_{v} / E_{g}^{2}-\delta_{\Delta} /\left(E_{g}+\Delta\right)^{2}\right] \partial_{z} h(z)$. Finally, we project this SO operator into the two lowest (spin-degenerate) eigenstates $|i\rangle_{\sigma_{z}}=\left|\vec{k}_{\|} i\right\rangle\left|\sigma_{z}\right\rangle,\left\langle\vec{r} \mid \vec{k}_{\|} i\right\rangle=$ $\exp \left(i \vec{k}_{\|} \cdot \vec{r}_{\|}\right) \varphi_{i}(z), i=e, o$, and $\sigma_{z}=\uparrow, \downarrow$, of the symmetric 
well $\left(H_{\mathrm{QW}}\right)$ (Fig. 1). This directly leads to the $\mathcal{H}$ in (1) with the SO coupling $\eta$ (2) [15]. Note that this new SO interaction is nonzero even in symmetric wells as $\eta$ arises from the coupling between the ground state (even) and the first excited state (odd) [Eq. (2)]. We can generalize $\mathcal{H}$ to include the Rashba $\alpha$ and the linearized Dresselhaus $\beta$ SO couplings. Next we determine the magnitude of $\eta$ (and $\alpha$, $\beta$ ) for realistic quantum wells with two subbands.

Self-consistent calculation of the SO couplings. - We consider modulation-doped quantum wells similar to those experimentally investigated in Ref. [16]. Our wells, however, have two occupied subbands. Similarly to Ref. [16], we study cases with constant chemical potentials [17]. By self-consistently solving Poisson and Schrödinger's equations we determine the energy levels $\varepsilon_{e}$, $\varepsilon_{o}$ and the confined wave functions $\varphi_{i}(z), i=e, o$ of the wells. We then calculate (i) $\eta$ via Eq. (2), (ii) $\alpha_{i}$ from equations similar to Eq. (2) for each subband, and (iii) $\beta_{i}=\gamma_{c}\left\langle i\left|k_{z}^{2}\right| i\right\rangle, \gamma_{c}$ is the bulk Dresselhaus SO parameter [18]. The structural symmetry of the wells and their charge densities can be changed via a gate potential $V_{b}$.

Our calculated SO couplings $\eta, \alpha_{i}$, and $\beta_{i}, i=e, o$, for an InAlAs/InGaAs/InAlAs single quantum- well ("sample 3" in [16]) are all comparable in magnitude [Fig. 2(a)]. Note that our $\alpha_{e} / \beta_{e}$ ratio is consistent with the experimental one in Ref. [19]. In addition, $\alpha_{e}$ vs $V_{b}$ here agrees well with the experimental data in Fig. 3 ("triangle up") of Ref. [16] (see also Fig. 4 in [20]). Our $\alpha_{i}$ [21] and $\beta_{i}$ are also consistent with those of Ref. [22]. Note that for the single well studied here $\eta$ does not vary appreciably with the gate $V_{b}$, similarly to $\beta_{i}$ and as opposed to $\alpha_{i}$.

For a double-well structure, on the other hand, we find that $\eta$ has a "resonant behavior," changing by about an order of magnitude as $V_{b}$ is swept across $V_{b}=0$ [Fig. 2(b)] (this may have a dramatic effect on Shubnikov-de Haas measurements). $V_{b}=0$ corresponds to a fully symmetric double well. In contrast to the single-well case, $\alpha_{e}$ and $\alpha_{o}$ have opposite signs and undergo abrupt changes in magnitudes near $V_{b}=0$ [Fig. 2(b), dashed lines]. Similarly to the single-well case, $\beta_{e}$ and $\beta_{o}$ are also essentially constant for a double well [Fig. 2(b), dotted lines]. A detailed account of our results will be presented elsewhere. Having established that the new SO coupling $\eta$ is sizable, in what follows we focus on a fully symmetric well to investigate physical effects arising solely from $\eta$.

Fully symmetric case: Eigensolutions. - Let us consider a two-subband well (single or double) described by the Hamiltonian $\mathcal{H}$ in (1) (we assume a negligible Dresselhaus term [19]). In the basis $\left\{|e\rangle_{\uparrow},|o\rangle_{\downarrow},|o\rangle_{\uparrow},|e\rangle_{\downarrow}\right\}$ $\mathcal{H}$ becomes

$$
\tilde{\mathcal{H}}=\left(\begin{array}{cccc}
\frac{\hbar^{2} k^{2}}{2 m^{*}}+\varepsilon_{e} & -i \eta k_{-} & 0 & 0 \\
i \eta k_{+} & \frac{\hbar^{2} k^{2}}{2 m^{*}}+\varepsilon_{o} & 0 & 0 \\
0 & 0 & \frac{\hbar^{2} k^{2}}{2 m^{*}}+\varepsilon_{o} & -i \eta k_{-} \\
0 & 0 & i \eta k_{+} & \frac{\hbar^{2} k^{2}}{2 m^{*}}+\varepsilon_{e}
\end{array}\right)
$$

Both the upper-left $(U)$ and lower-right $(L)$ blocks of $\tilde{\mathcal{H}}$ have eigenvalues

$$
\varepsilon_{ \pm}(\vec{k})=\epsilon_{k} \pm \hbar \Omega,
$$

with $\epsilon_{k}=\hbar^{2} k^{2} / 2 m^{*}+\epsilon_{+},(\hbar \Omega)^{2}=\eta^{2} k^{2}+\epsilon_{-}^{2}$, and eigenvectors

$$
\begin{aligned}
\left|\psi_{1}\right\rangle_{+}^{U} & =\sin (\theta / 2)|e\rangle_{\uparrow}+\cos (\theta / 2) e^{i \phi}|o\rangle_{\downarrow}, \\
\left|\psi_{2}\right\rangle_{+}^{L} & =\cos (\theta / 2)|o\rangle_{\uparrow}+\sin (\theta / 2) e^{i \phi}|e\rangle_{\downarrow}, \\
\left|\psi_{3}\right\rangle_{-}^{U} & =\cos (\theta / 2)|e\rangle_{\uparrow}-\sin (\theta / 2) e^{i \phi}|o\rangle_{\downarrow}, \\
\left|\psi_{4}\right\rangle_{-}^{L} & =\sin (\theta / 2)|o\rangle_{\uparrow}-\cos (\theta / 2) e^{i \phi}|e\rangle_{\downarrow} .
\end{aligned}
$$

Here, $e^{i \phi}=\left(-k_{y}+i k_{x}\right) / k, \cos (\theta)=1 / \sqrt{1+\left(\eta k / \epsilon_{-}\right)^{2}}$, and $\vec{k}=k(\sin \phi,-\cos \phi)$ (here we drop the " $\|$ " in $\vec{k}_{\|}$). For $\eta k \ll 2 \epsilon_{-}$we can expand $\varepsilon_{ \pm}(\vec{k})$ in (8) and define effective masses $m_{ \pm}^{*}=m^{*} /\left[1 \pm 2 \varepsilon_{\mathrm{SO}} / \epsilon_{-}\right]$, where $\varepsilon_{\mathrm{SO}}=$ $\eta^{2} m^{*} / 2 \hbar^{2}$ is the energy scale of the new SO coupling. For the double well of Fig. 2(b), $m_{-}^{*}$ is reduced by $\sim 5 \%$ compared to the bulk value $m^{*}$. This could be measured via, e.g., cyclotron-resonance experiments [23].

Novel Zitterbewegung. - The dynamics of electron wave packets in wells with SO interaction exhibit an oscillatory motion [7]- the Zitterbewegung. For our new SO interaction, a wave packet $|\chi\rangle$ moves according to $\left\langle\chi\left|\vec{r}_{H}(t)\right| \chi\right\rangle$ where $\vec{r}_{H}(t)=U^{\dagger} \vec{r} U$ is the position operator in the Heisenberg picture $[U=\exp (-i \mathcal{H} t / \hbar)]$ with components

$$
\begin{aligned}
x_{H}(t)= & \mathbf{1} \otimes \mathbf{1} x(0)+\mathbf{1} \otimes \mathbf{1} \frac{p_{x}}{m^{*}} t+\frac{\eta}{\hbar} t \tau^{x} \otimes \sigma^{y} \\
& -\frac{\eta}{2(\hbar \Omega)^{2}}\left[\boldsymbol{\epsilon}_{-} \tau^{y} \otimes \sigma^{y}+\frac{\eta}{\hbar} p_{y} \mathbf{1} \otimes \sigma^{z}\right] \\
& \times[\cos (2 \Omega t)-1]+\frac{\eta}{2(\hbar \Omega)^{3}}\left[\boldsymbol{\epsilon}_{-}^{2} \tau^{x} \otimes \sigma^{y}\right. \\
& \left.+\epsilon_{-} \frac{\eta}{\hbar} \tau^{z} \otimes \mathbf{1} p_{x}+\left(\frac{\eta}{\hbar}\right)^{2} p_{y} \tau^{x} \otimes\left(p_{x} \sigma^{y}+p_{y} \sigma^{x}\right)\right] \\
& \times[\sin (2 \Omega t)-2 \Omega t],
\end{aligned}
$$

and $y_{H}(t)$, obtained from Eq. (13) via the replacements $\left(p_{x}, \sigma^{x}\right) \mapsto\left(p_{y}, \sigma^{y}\right),\left(p_{y}, \sigma^{y}\right) \mapsto\left(-p_{x},-\sigma^{x}\right)$ (i.e., a $\pi / 2$ rotation about the $z$ axis). Similar expressions can be derived for the spin components $\sigma_{H}^{i}(t), i=x, y, z$ [24].

For simplicity, we evaluate the expectation value of $\vec{r}_{H}(t)$ for planes waves ("wide wave packets"). For a spin-up electron injected into the lowest subband along the $y$ axis with (group) velocity $\vec{v}_{g}=\left(\hbar k_{0 y} / m^{*}\right) \hat{y}$, we find

$$
\begin{gathered}
\left\langle x_{H}(t)\right\rangle=\frac{\eta^{2} k_{0 y}}{2(\hbar \Omega)^{2}}[1-\cos (2 \Omega t)], \\
\left\langle y_{H}(t)\right\rangle=\frac{\hbar k_{0 y}}{m^{*}} t+\frac{\eta^{2} k_{0 y} \epsilon_{-}}{2(\hbar \Omega)^{3}}[\sin (2 \Omega t)-2 \Omega t],
\end{gathered}
$$

assuming $x(0)=y(0)=0$. Equations (14) and (15) show that cycloidal motion is possible in our system. This differs 
qualitatively from the Rashba SO Zitterbewegung which is always perpendicular to the initial $\vec{v}_{g}$.

Figure 3 shows trajectories for three distinct $\vec{v}_{g}=\left(\hbar k_{0 y} / m^{*}\right) \hat{y}$-all with $k_{0 y}>0$. We find motion $o p$ posite to and along the $y$ axis (orbits I and III, respectively) and even a closed path (II). To understand this behavior we note that for $\eta k_{0 y} \ll \varepsilon_{o}-\varepsilon_{e}$ the linear-in- $t$ terms in $\left\langle y_{H}(t)\right\rangle$ can be recast into $\hbar k_{0 y} t / m_{-}^{*} \Rightarrow$ the injected wave moves with the renormalized velocity $v_{g}^{*}=\hbar k_{0 y} / m_{-}^{*}$. Hence, for orbit I, $\mu<1 \Rightarrow m_{-}^{*}<0$ [25] and $v_{g}^{*}<0$, for orbit II, $\mu=1 \Rightarrow m_{-}^{*} \rightarrow \infty$ and $v_{g}^{*}=0$, and for orbit III, $\mu>1 \Rightarrow m_{-}^{*}>0$ and $v_{g}^{*}>0$. Though remarkable, we stress that the orbits I and II occur for unusual parameters (e.g., $\varepsilon_{F}<\varepsilon_{\mathrm{SO}} / 10$ ). However, these orbits do show that our SO Hamiltonian has a physical mechanism allowing for cyclotronic motion without magnetic fields.

Spin-Hall conductivity $\sigma_{x y}^{z}$. - The spin-Hall effect is a convenient probe for SO effects in wells [26]. We have calculated $\sigma_{x y}^{z}$ ("clean limit") in the presence of an external magnetic field $B$ by following the approach of Rashba [27], which allows us to properly account for both the intrabranch and interbranch contributions in the Kubo formula [28]. Here we focus on the $B \rightarrow 0$ limit where we find $\sigma_{x y}^{z}=0$ for $\varepsilon_{F}>\varepsilon_{o}$ (two subbands occupied) and

$$
\sigma_{x y}^{z}=\frac{e}{8 \pi}\left[\frac{1}{\kappa_{1}}\left(\frac{1}{\kappa_{3}}-2\right)+\frac{\kappa_{2}+\kappa_{1} / 2}{2 \kappa_{3}^{3}}\right]
$$

for $\varepsilon_{e}<\varepsilon_{F}<\varepsilon_{o}$ (upper subband empty), where $\kappa_{1}=2 \varepsilon_{\mathrm{SO}} / \epsilon_{-}, \quad \kappa_{2}=\left(\varepsilon_{F}-\epsilon_{+}\right) / 2 \epsilon_{-}, \quad$ and $\quad \kappa_{3}=$ $\sqrt{\kappa_{1}^{2} / 4+\kappa_{1} \kappa_{2}+1 / 4}$. Note that $\sigma_{x y}^{z}$ is nonzero and nonuniversal in this range, shows a discontinuity at $\varepsilon_{F}=\varepsilon_{o}$, and changes sign as a function of $\varepsilon_{F}$. Details of our calculation of $\sigma_{x y}^{z}$ and a thorough discussion will be presented elsewhere [29]. Here we just note that measurements of $\sigma_{x y}^{z}$ (versus $\varepsilon_{F}$ ) in symmetric two-subband wells offer a possibility to probe our new SO interaction [26]. Note that the Rashba and the linearized Dresselhaus spinHall conductivities are identically zero in the de limit $[27,30]$.

We have introduced an intersubband-induced SO interaction in quantum wells with two subbands. The corresponding SO coupling $\eta$ (whose magnitude is similar to Rashba coupling) is nonzero even in symmetric wells. This new SO interaction gives rise to a nonzero spin-Hall conductivity, renormalizes the bulk mass by $\sim 5 \%$ (measurable via cyclotron resonance [23]) in double wells, and can induce a cycloidal Zitterbewegung. Weak antilocalization $[20,31]$ should offer another possibility to measure $\eta$.

We thank S. Erlingsson, D.S. Saraga, D. Bulaev, J. Lehmann, M. Duckheim, L. Viveiros, G. J. Ferreira, R. Calsaverini, and E. Rashba for useful discussions. This work was supported by the Swiss NSF, the NCCR Nanoscience, DARPA, ONR, CNPq, FAPESP, DFG via SFB 689, and U.S.A. NSF PHY99-07949.
[1] Semiconductor Spintronics and Quantum Computation, edited by D. D. Awschalom, D. Loss, and N. Samarth (Springer, Berlin, 2002); I. Zutic et al., Rev. Mod. Phys. 76, 323 (2004).

[2] G. Dresselhaus, Phys. Rev. 100, 580 (1955).

[3] M.I. Dyakonov and V.Y. Kachorovskii, Sov. Phys. Semicond. 20, 110 (1986); G. Bastard and R. Ferreira, Surf. Sci. 267, 335 (1992).

[4] Y.A. Bychkov and E.I. Rashba, J. Phys. C 17, 6039 (1984).

[5] G. Engels et al., Phys. Rev. B 55, R1958 (1997); J. Nitta et al., Phys. Rev. Lett. 78, 1335 (1997).

[6] J. Schliemann et al., Phys. Rev. Lett. 90, 146801 (2003); M. Trushin and J. Schliemann, Phys. Rev. B 75, 155323 (2007).

[7] J. Schliemann et al., Phys. Rev. Lett. 94, 206801 (2005).

[8] I. Vurgaftman et al., J. Appl. Phys. 89, 5815 (2001).

[9] E. O. Kane, J. Phys. Chem. Solids 1, 249 (1957).

[10] Note that $\delta_{v}=E_{g}^{b}-E_{g}-\delta_{c}$ and $\delta_{\Delta}=\delta_{v}-\Delta_{b}+\Delta$, where $E_{g}^{b}$ and $\Delta_{b}$ are, respectively, the fundamental and split-off band gaps in the barriers and $\delta_{c}$ the conductionband potential offset [see, e.g., P. Pfeffer and W. Zawadzki, Phys. Rev. B 68, 035315 (2003)].

[11] R. Lassnig, Phys. Rev. B 31, 8076 (1985).

[12] T. Darnhofer and U. Rössler, Phys. Rev. B 47, 16020 (1993).

[13] In (5) we neglect an effective Darwin term as it arises in relativistic quantum mechanics. This term leads to a rigid intraband shift and can be absorbed into $\varepsilon_{e}, \varepsilon_{o}$.

[14] We treat $m^{*}$ as a parameter in our model (the Kane effective mass neglects corrections from higher-lying bands).

[15] In Eq. (1) we assume that $\left\langle e\left|\partial_{z} V\right| o\right\rangle$ and (in turn) $\eta$ are real. If $\eta=|\eta| e^{i \delta}$, we have to replace $\tau^{x}$ by $\cos (\delta) \tau^{x}-$ $\sin (\delta) \tau^{y}$ in (1). The freedom in fixing $\delta$ has no physical consequences since a change in $\delta$ can be compensated by applying the phase factor $\exp \left(i \delta \tau^{z}\right)$ to the basis states: $\left[\cos (\delta) \tau^{x}-\sin (\delta) \tau^{y}\right] \exp \left(i \delta \tau^{z}\right)=\tau^{x}$. Thus, $\mathcal{H}$ enjoys a $\mathrm{U}(1)$ gauge symmetry corresponding to a rotation about the $z$ direction in the $\vec{\tau}$ space.

[16] T. Koga et al., Phys. Rev. Lett. 89, 046801 (2002).

[17] Wells with constant areal densities yield similar results in the parameter range studied.

[18] J.-M. Jancu et al., Phys. Rev. B 72, 193201 (2005).

[19] S. Giglberger et al., Phys. Rev. B 75, 035327 (2007).

[20] T. Koga et al., Phys. Rev. B 74, 041302(R) (2006).

[21] C. M. Hu et al. [Phys. Rev. B 60, 7736 (1999)] find a larger Rashba constant in the second subband.

[22] E. Shafir et al., Phys. Rev. B 70, 241302(R) (2004).

[23] H. K. Ng et al., Appl. Phys. Lett. 75, 3662 (1999).

[24] E. Bernardes et al., Phys. Status Solidi C 3, 4330 (2006).

[25] L. Sena et al., Phys. Rev. B 72, 235309 (2005).

[26] V. Sih et al., Nature Phys. 1, 31 (2005).

[27] E. I. Rashba, Phys. Rev. B 70, 201309(R) (2004).

[28] J. Sinova et al., Phys. Rev. Lett. 92, 126603 (2004).

[29] M. Lee et al. (to be published).

[30] J. Sinova et al., Solid State Commun. 138, 214 (2006).

[31] V. A. Guzenko et al., Phys. Status Solidi C 12, 4227 (2007). 